\title{
A new Frontier: Nano-Science and Engineering
}

\author{
Sanjita Lamichhane $\ddagger$ and S K Lamichhane $†$ \\ ${ }^{\ddagger}$ Tribhuvan University, IOE, WRC, Pokhara, and ${ }^{\dagger}$ Prithwi Narayan Campus, Pokhara, sklamichhane@hotmail.com
}

Swirling galaxy denoting the cosmos and massive accelerators used to probe matter at successively smaller scales: from the atom down to the quark and beyond. Such conventional frontier of physics is largely concerned with reductionism. The notion, that once we know the laws of nature that operates on the smallest possible scales, the mysteries of the universe will be revealed to us.

Human quest for understanding of the natural world leads to scientific theories. Physics is the science developed to study of matter and energy and tries to answer basic questions about the world/universe, how it is put together and how it changes. Some physicist called 'experimental physicist' perform fully designed experiment and then compare their results with what was predicted to happen. Such predictions come to laws and theories. Coherent theories insist to generate technology [1]. So, advances in basic science and creation of new technology are closely related. In many cases, there is a deep symbiosis between discovery in science and technology. Nanoengineering is a multi-disciplinary engineering field which combines the areas such as materials science and engineering, chemistry, physics, biology and medicine. Indeed, it is all about generating new solutions based on atomic and molecular scale manipulations.

Engineering might lead directly to the smallest and fastest, strongest and lightest materials ever made [2]. Thus, Nanotechnology is the creation of new materials, devices and systems at molecular level where phenomenon associated with atomic and molecular interactions strongly influence macroscopic material properties with significantly improved mechanical,optical, chemical and electrical properties. A technology could exploit such physical properties and hence resulted into instrumentation. As for example, magnetic resonance imaging (MRI) is widely regarded as the advanced technique in medical diagnosis [3] since the discovery of $\mathrm{x}$-rays. MRI was made possible by research on nuclear resonance in chemical and biological systems, the development of super conducting magnets, theoretical research on tomography and image reconstruction, advances in numerical processing devices and the discovery of NMR.

In December 1959, Nobel Laureate R Feymenn deliver a visionary talk entitled "There is plenty of room at the bottom" on the occasion of American Physical Society's meeting at California Institute of Technology. That particular speech was a defining moment of "Nanotechnology" long before anything 'nano' appears on the horizon. Nanotechnology commonly refers to the fabrication, study, and manipulation of structures having sizes in the range from one to one hundred nanometers (a nanometer is a billionth of a meter). This length scale bridges the important gap between atoms and molecules (which are less than one nanometer in size) and bulk materials; requiring fundamental chemistry and quantum physics. To develop this new cluster of technologies, there is an acute need for highly trained personnel with good understanding of the natural laws that govern the workings of not only atoms and molecules but also natural or manufactured nanoscopic and mesoscopic structures and systems (e.g., clusters, fullerenes, nanotubes, macromolecules, nanorobots, and nanosystems etc). The ability to control matter at nanoscale allows us to take advantage of phenomena which predominate at these length scales, leading to the production of novel materials and devices exhibiting qualitatively different properties and functions than those of the corresponding bulk materials.

Immediate applications of nanostructures and nano-devices include quantum electronic devices, bio-surgical instruments, microelectrical sensors, functionally graded materials, and many others with great promises for commercialization. The field is loosely divided into four subareas: micro and nanoinstruments, nanoelectronics, nanobiosystems, and nanoengineered materials. The 
first addresses some of the most far-reaching yet practical applications of miniature instruments for measuring atoms or molecules in chemical, clinical, or biochemical analysis; in biotechnology for agent detection; and environmental analysis. The second category, nanoelectronics, concerns the development of systems and materials required for the electronics industry to go beyond current technological limits - producing even finer detail than features in a high-performance microprocessor chip. Also in this category is a new generation of electronics based on plastics, which is expected to create new markets with applications ranging from smart cards to tubelike computers. The third class, nano-biosystems, can be described as molecular manipulation of biomaterials and the associated miniaturization of analytical devices such as DNA, peptide, protein, and cell chips. The last subarea, nanoengineered materials, looks at several classes of advanced materials including nanocrystalline materials and nanopowders used in electronics and photonics application, as catalysts in automobiles, in the food and pharmaceutical industries, as membranes for fuel cells, and for industrial-scale polymers.

Actually, nanotechnology represents a convergence in the bottom up and top-down approaches to fabrication into a common field that share complementary expertise and methodology. An example of this is concurrent development of the analytical characterization techniques that allow researchers to view things down to the atomic scale, in particular the atomic force, scanning tunneling and electron microscopes. Fig. belongs to 2D micrograph micromechanics taken by atomic force microscope (AFM), is one of the key instruments in nanotechnology. It is the tool by which it can be possible to move atoms and molecules around in a controlled fashion so that atomic manipulation is possible. A portable AFM has also been developed at IBM recently.

Materials made in this size range (between molecules and the corresponding bulk materials) often have size-dependent properties. The best known example is the ever decreasing size of silicon-based integrated circuit components. Moore, the founder of Intel, predicted in 1965 that these components would double in density approximately in every two years. The size of silicon-based transistor as a function of time progresses from 10 micrometre in the 1970's to $45 \mathrm{~nm}$ currently. Nanotechnology engineering is not only key to decreasing the fabrication scale, but also solving device performance issues through improved design that will become increasingly more important in reduced scale.

The development of very large-scale integrated (VLSI) circuits used for information processing depends on reducing the size of the individual circuit component down to the nanoscale [4]. But, nanotechnology brings its own distinctive challenges. Firstly, handling matter at the atomic scale (nanotechnology is all about a "atomically precise engineering") and secondly, in order for atomically precisely engineered objects to be useful for humans, they need to be somehow multiplied, which introduces the problem of handling vast numbers of entities with different behavior. And lastly, continuing rapid evolution of nanotechnology,

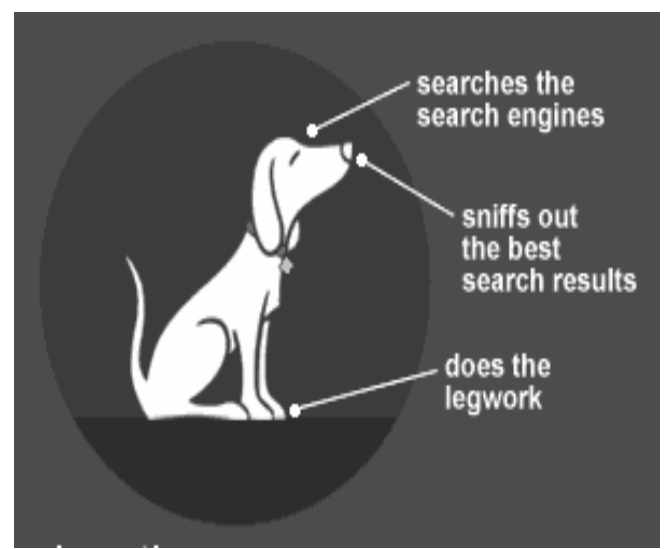

An overview of nanotech
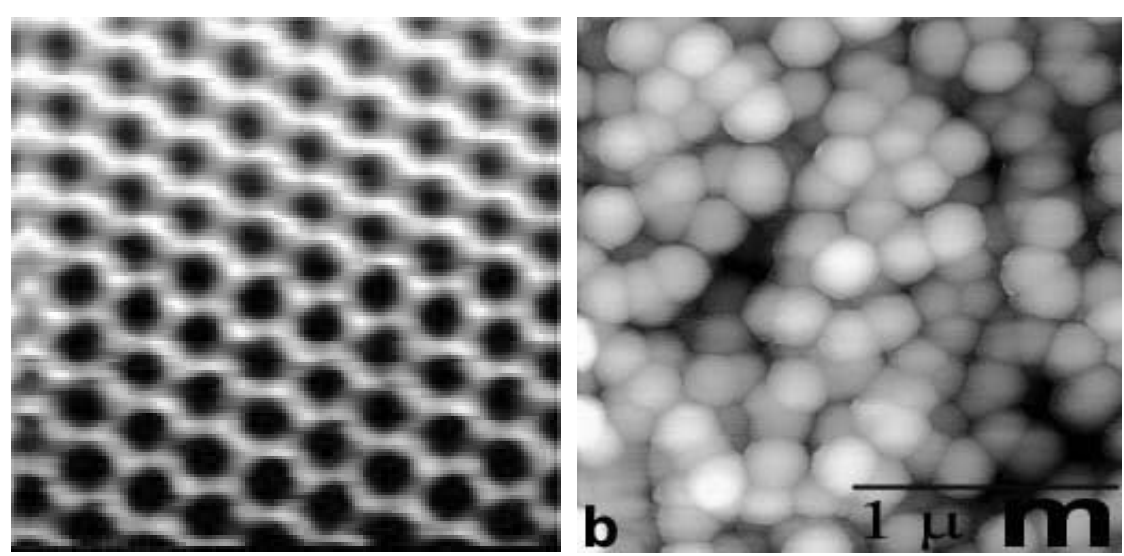

Graphite and Silicon in atomic resolution as observed by AFM 
one should not underestimate the multidisciplinary nature (it uses techniques from, physics, chemistry, bioscience, material science, polymer science and engineering) of nanotechnology. Many active fields in its research demand an understanding of diverse areas of science. Sometimes this problem is solved by assembling teams of researchers but members of the team still need to be able to effectively communicate with one another.

Make small is the technological edict that has changed the world. But, the idea of making 'nanostructures' that comprise just one or a few atoms has get appeal both as scientific challenge and practical reasons [5]. The structure and the size of an atom represents a fundamental limits to make anything smaller would require manipulating atomic nuclei essentially transmuting one chemical element into another. In recent years, scientists have learned various techniques for building nanostructures. But they have only just begins to investigate their properties and potential applications. The age of nanofabrication and nanoscience has dawned, but the age of nanotechnology, finding practical uses of nanostructures has not really started [6]. At its maturity level we can say " Nanotech will have an impact equal to the Industrial Revolution" [7]. Thus, nanotechnology is widely considered to constitute the basis of the next technological revolution, following on from the first Industrial Revolution, which began around 1750 with the introduction of the steam engine and steelmaking. The Industrial Revolution constituted as profound a change in society and civilization as the earlier Stone, Bronze and Iron revolutions, each of which ushered in a distinctly new age in the history of human civilization.

The branch of mechanics research in this emerging field is termed as nano/micro-mechanics, both scientifically rich and technologically significant, of solids as a distinct and unifying theme [8]. The presentation at workshop and the open discussion revealed emergence of a range of interesting lines of investigation builtin nano-mechanics, havepotentials to microelectronics, information technology, biotechnology. The potentials of nanotechnology will be enhanced, in particular, by exploring the nano/micro- mechanics of coupled phenomena as shown in figure above and of multi-scale phenomena. Examples of coupled phenomena include modification of quantum states of materials caused by mechanical strains, ferroelectric transformations induced by electric field and mechanical stresses, chemical reaction processes biased by mechanical stresses, and change of biomolecular conformity of proteins caused by environmental mechanical strain rates. Multi-scale phenomena arise in situations where properties of materials to be exploited in applications at a certain size scale are controlled by physical processes occurring on size scale whose orders of magnitude is smaller. Important problems of this kind arises, for example, in thermo-mechanical behavior of thinfilm nanostructures, evolution of surface and bulk nanostructures caused by various material defects, nano-indentation, and nano-tribological responses of solids. A commitment to nano/micro-mechanics will lead to a strong foundation of understanding and confidence underlying the capabilities in modeling and experimentation embodying a high degree of rigor [9].

Research in the area of nanoscale science and engineering (NSE) is aimed at supporting high risk/high reward, long-term nanoscale science and engineering research leading to potential breakthroughs in areas such as materials and manufacturing, nanoelectronics, medicine and healthcare, environment and energy, chemical and pharmaceutical industries, biotechnology and agriculture, computation and information technology, improving human performance and national security. It also addresses the development of a skilled workforce in this area as well as the ethical, legal and social implications of future nanotechnology. In US, NSE initiative focuses on seven high risk/high reward research areas, where special opportunities exist for fundamental studies in synthesis, processing, and utilization of NSE. These seven areas are: biosystems at the nanoscale and nanostructure, novel phenomena and quantum control, device and system architecture, nanoscale processes in the environment, modeling and simulation at the nanoscale, manufacturing processes 
at the nanoscale; and societal and educational implications of scientific and technological advances in nanoscale. Governments in developed countries have take initiatives and boosted up their research funding in nanotechnology. Between 1997 and 2005, investment in nanotech research and development by governments around the world soared from $\$ 432$ million to about $\$ 4.1$ billion [11], and corresponding industry investment exceeded that of governments. By 2015, products incorporating nanotech will contribute approximately $\$ 1$ trillion to the global economy. About two million workers will be employed in nanotech industries [11], and three times that many will have supporting jobs.

In conclusion, unlike the computer science in $1960 \mathrm{~s}$ and biotechnology in 1980s, nanotech is getting towards maturity rapidly. There are tremendous opportunities for synthesizing nanostructured materials with new architectures. Synthesis can be done from the assembly of atomic layers and clusters and by myriad of other techniques now becoming available, such as nanolithography, biological templating etc. The keys to the future of nanostructured material, however, are our ability to continue to improve the properties of materials by artificially structuring them on nanometer length scale and our ability to develop the methods for producing these materials in commercially viable quantities. The challenge is how can we contribute to this broad-base and diverse research agendas? Mechanics and material engineering are really two sides of a coin, closely integrated and related. For the last decade, cooperative effort has started in it and resulted for better understanding and synthesizing of materials and structures a little. Across the uncertainty in time and space, expertise and funding, system integration is not yet attainable at the level of satisfaction [10]. Based on what we have learned to date about mesoscopic physics and on the success secured by the entry of nanostructure devices into industry, field of nanoscience and engineering have wide future.

\section{References}

[1]. Asif, S. A. S., Wahl, K. J., Colton, R. J., and Warren, O. L. 2001. Quantitative Imaging of nanoscale Mechanical Properties Using Hybrid Nanoindentation and Force Modulation. J. Appl. Phys. 90, 5838.

[2]. Boresi, A. P. and Chong, K. P. 2000. Elasticity in Engineering Mechanics, John Wiley, New York.

[3]. Boresi, A. P., Chong, K. P. and Saigal, S. 2002. Approximate Solution Methods in Engineering Mechanics, John Wiley, New York.

[4]. Bhushan, B., Kulkarni, A.V., Banin, W., and Wyrobek, J. T. 1996. Nanoindentation and

Picoindentation Measurement Using a Capacitive Transducer System in Atomic Force

Microscopy.

[5]. Proc. NATO Adv. Res. Workshop on Smart Structures, held in Pultusk, Poland, 6/98, Smart Structures, Kluwer Academic Publ. 1999, pp 37.

[6]. Chong, K. P., 2003. "Nano-scale Mechanics Research and Challenges", Plenary Paper, Study of Matter at Extreme Conditions Conference, Miami Beach, FL.

[7]. Katti, K. S., Katti, D. R., Tang, J., and Sarikaya, M. 2000. Proc. Mat. Res. Soc., Symp. AA.

[8]. Monteiro P. J. M., Chong, K. P., Larsen-Basse, J. and Komvopoulos, K., Eds. 2001. Long-term Durability of Structural Materials, Elsevier, Oxford, UK.

[9]. VanLandingham, M. R., Villarrubia, J. S., Guthrie, W. F, and Meyers, G. F. 2001. Nanoindentation of Polymers: An Overview Macromolecular Symposia 167: Advances in SPM of Polymers,

[10]. V. V. Tsukruk and N. D. Spencer, Wong, E. 1996. An Economic Case for Basic

Research. Nature 381, 187.

[11]. http://www.nanotech.uwaterloo.ca/what_is_Nano/ 